\title{
Microgravity and the respiratory system
}

\author{
G. Kim Prisk ${ }^{1,2}$
}

\author{
Number 5 in the Series "Physiology in Respiratory Medicine" \\ Edited by R. Naeije, D. Chemla, A. Vonk-Noordegraaf and A.T. Dinh-Xuan
} Affiliations: ${ }^{1}$ Dept of Medicine, University of California, San Diego, CA, and ${ }^{2}$ Dept of Radiology, University of
California, San Diego, CA, USA.

Correspondence: G.K. Prisk, Depts of Medicine and Radiology, University of California, San Diego, 9500 Gilman Drive, La Jolla, CA 92093-0852, USA. E-mail: kprisk@ucsd.edu

ABSTRACT The structure of the lung, with its delicate network of airspaces and capillaries, means that gravity has a profound influence on its function. Studies of lung function in the absence of gravity provide valuable insight into how, for we Earth-bound individuals, its unavoidable effects shape our lung function. Gravity causes uneven ventilation in the lung through the deformation of lung tissue (the so-called Slinky effect), and uneven perfusion through a combination of the Slinky effect and the zone model of pulmonary perfusion. Both ventilation and perfusion exhibit persisting heterogeneity in microgravity, indicating important other mechanisms. However, gravity serves to maintain a degree of matching of these two processes, so that the ventilation/perfusion ratio, and thus gas exchange, remains efficient. Therefore, while both ventilation and perfusion are more uniform in spaceflight, gas exchange is seemingly no more efficient than on Earth. Despite the changes in lung function when gravity is removed, the lung continues to function well in weightlessness. Unlike many other organ systems, the lung does not appear to undergo structural adaptive changes when gravity is removed, and so there is no apparent degradation in lung function upon return to earth, even after 6 months in space.

\section{@ERSpublications}

The structure of the lung means that gravity has a profound influence on its function http://ow.ly/sZEZt

Previous articles in this series: No 1: Naeije R, Vachiery J-L, Yerly P, et al. The transpulmonary pressure gradient for the diagnosis of pulmonary vascular diseases. Eur Respir J 2013; 41: 217-223; No 2: Hughes JMB, van der Lee I. The TL,NO/ TL,CO ratio in pulmonary function test interpretation. Eur Respir J 2013; 41: 453-461. No. 3: Vonk-Noordegraaf A, Westerhof N. Describing right ventricular function. Eur Respir J 2013; 41: 1419-1423; No. 4: Hamzaoui O, Monnet X, Teboul J-L. Pulsus paradoxus. Eur Respir J 2013; 42: 1696-1705.

Received: Jan 022014 | Accepted after revision: Jan 212014 | First published online: March 62014

Support statement: G.K. Prisk is supported by US National Institutes of Health grant R01-HL104117 and by the National Space Biomedical Research Institute though National Aeronautics and Space Administration (NASA) co-operative agreement NCC 9-58. Many of the studies were performed under contracts and grants from NASA.

Conflict of interest: None declared.

Copyright $\odot$ ERS 2014 


\section{Introduction}

Given that very few of us will ever experience sustained periods in the absence of gravitational effects, one might reasonably ask why even write an article about the effects of removing gravity on the lung? The answer is that of all the organs in the body, the lung is, by the nature of its structure, the most distensible. At functional residual capacity (FRC), the lung is comprised of something in the vicinity of $\sim 1 \mathrm{~L}$ of tissue and blood and $\sim 3 \mathrm{~L}$ of air, giving it an overall low density ( $\sim 25 \%$ of that of the most other organs in the body). While this low density might argue for a limited effect of gravity, the intricate structure of the lung with the numerous air-tissue interfaces of the alveoli makes for a highly compliant structure that deforms under its own weight. Thus, the normal lung is markedly affected by the ever-present gravity we all experience and these effects directly influence virtually every measurement of lung function that we make. For that reason alone, understanding how gravity affects the lung is a relevant topic, and just as we might place someone in a hot or cold environment in order to study thermoregulation, altering the level of gravity the lung experiences provides a means to study gravitational effects.

\section{Finding zero gravity}

Altering gravity itself is not practical in the true sense of the word. Doing so would require altering the mass of the Earth. Rather, when the term "altered gravity" is used, what is actually being referred to is an alteration in the inertial forces we experience, which are themselves indistinguishable from gravity. We have all experienced this is in a lift in a high-rise building, which, as it accelerates upwards at the beginning of the journey, gives the feeling that we are suddenly heavier. Similarly, as it decelerates upon arrival at an upper floor, we transiently feel lighter. What we are sensing is the additional inertial forces from the acceleration on top of a constant gravitation force.

We usually refer to these as altered $g$-levels, where $1 \times g$ is an acceleration of $9.81 \mathrm{~m} \cdot \mathrm{s}^{-2}$, the acceleration due to gravity on the Earth's surface. Raising the $g$-level is relatively easy and almost always accomplished by simply moving in a curved trajectory, typically in a centrifuge where the circular path of the centrifuge arm results in a centripetal acceleration dependent on the angular velocity and the radius. Lowering the $g$-level is, however, much more difficult. Contrary to some popular misconceptions, there are no zero-gravity rooms available. On the ground, the only possibility for low- $g$ comes from very short-lived transient accelerations in an elevator, a model that has been used for study [1], or in drop towers, the latter providing a few seconds of zero gravity for small samples. Longer periods of zero gravity require sustained periods of acceleration towards the centre of the Earth at $-1 \times g\left(-9.81 \mathrm{~m} \cdot \mathrm{s}^{-2}\right)$. This is achievable either in an aircraft that accelerates towards the ground at that rate or in orbital flight in a spacecraft. In the former case, the longest periods of zero gravity can be achieved by starting the manoeuvre with the aircraft ascending and ending with it descending, all the while maintaining a zero-gravity condition in the cabin. In essence, the aircraft follows a parabolic trajectory, just like a rock thrown upwards. In a typical aircraft (such as those used for commercial flight), periods of 20-25 s of zero gravity can be achieved, although these periods are "sandwiched" between periods of hypergravity $(\sim 1.8 \times \mathrm{g})$ that are necessary to fly the manoeuvre (see the review by KARMALI and SHELHAMER [2] for a detailed explanation of parabolic flight). Longer periods have been achieved using aircraft capable of supersonic speeds [3]. Sustained zero gravity can only be achieved in orbital or interplanetary flight. A spacecraft in orbit "falls" towards the centre of the Earth but, because of its forward velocity, continuously misses the Earth (thus staying in orbit), providing a continuous period of zero gravity. Despite the popular misconception that the spacecraft is so far above the Earth's surface as to be free of the Earth's gravitation influence (in low-Earth orbit, the acceleration due to gravity is still $>90 \%$ that on the surface), the zero gravity in the cabin is a result if the inertial forces of orbital motion cancelling the gravitational pull of the Earth. In this context, the old term "free fall" is, in fact, more descriptive of the situation. Furthermore, if an object is not at the centre of mass of the spacecraft, then very small residual accelerations exist, and for this reason, rather than the term zero gravity, the term microgravity is used. Overall, "weightlessness" may be the simplest descriptor.

\section{Underlying concepts}

To provide a framework for interpreting the results from microgravity studies of the lung, it is useful to briefly review two underlying concepts.

The zone model

The zone model of pulmonary perfusion is long established, dating back to the 1960s [4, 5]. Because of the low perfusion pressures in the pulmonary circulation, hydrostatic pressure differences in the lung, which are a direct result of gravity, are important in determining pulmonary perfusion. Moving from whatever part of the lung is lowermost (a posture-dependent condition) to the uppermost part, both pulmonary arterial and pulmonary venous pressures fall, in equal amounts. However, alveolar pressure does not and is equal in all 
a)

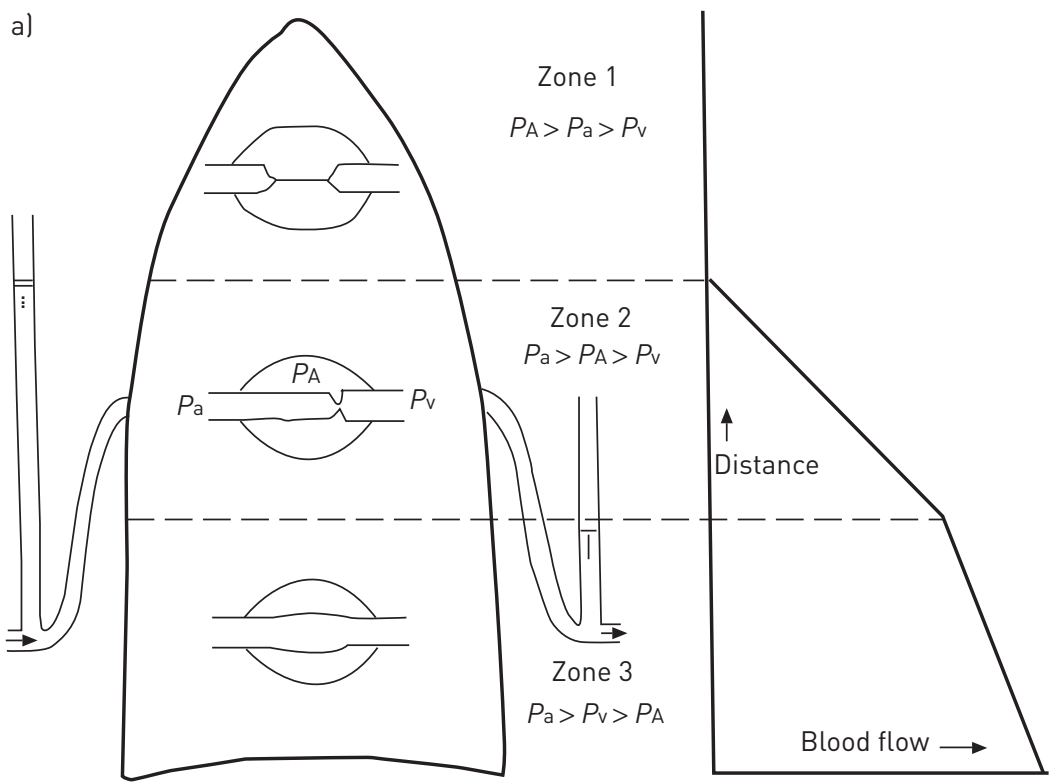

b)

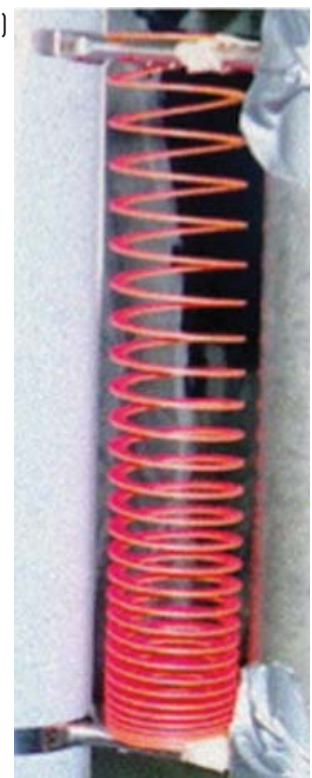

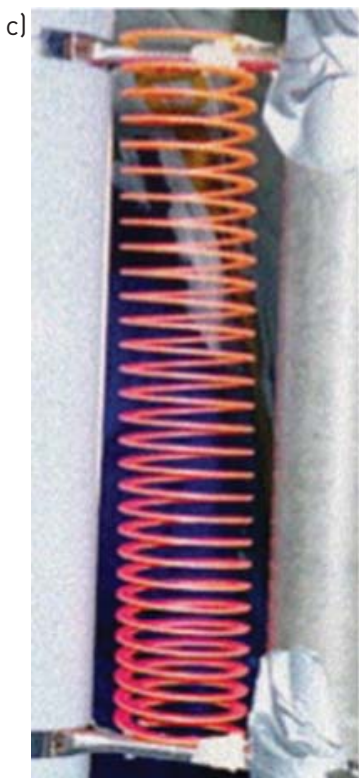

FIGURE 1 The two key concepts of the effects of gravity on the lung. a) The zone model of pulmonary perfusion. Regional perfusion depends on the relative values of pulmonary arterial pressure $(P \mathrm{a})$, pulmonary venous pressure $\left(P_{\mathrm{v}}\right)$ and alveolar pressure $\left(P_{\mathrm{A}}\right)$. In zone $1, P_{\mathrm{A}}$ exceeds both vascular pressures and there is no flow. In zone 3, both vascular pressures exceed $P_{\mathrm{A}}$ and so flow is determined by the arterial-venous pressure difference. Between these is zone 2 , in which $P_{\mathrm{v}}$ (but not $P$ a) is less than $P$ A, forming a Starling resistor effect in which flow is determined by the arterial-alveolar pressure difference. As $P$ A does not vary with height, there is a steep increase in perfusion moving down the lung. Reproduced from [5] with permission from the publisher. b) A Slinky spring fixed at the top and bottom under the effects of gravity. Note the deformation of the spring due to self-weight. c) The same spring in the absence of gravity. The spring is now uniformly expanded. The two pictures were taken by the author under conditions of $\sim 1.8 \times g$ and $\sim 0 \times g, \sim 45 \mathrm{~s}$ apart during parabolic flight.

parts of the lung (assuming patent airways). As shown in figure 1a, in the most gravitationally dependent lung, blood flow depends on the pressure difference between the arterial and venous sides of the pulmonary vasculature, a situation with which we are all familiar and comfortable. Blood flow varies little with height in this region, with a small increase as one moves lower, generally attributed to distension of the pulmonary capillaries as pressures rise. This is termed zone 3. In contrast, at the very uppermost portion of the lung, the low pulmonary vascular pressures coupled with hydrostatic effects can lead to pressures falling below alveolar pressure, and since the pulmonary capillaries are thin-walled and unsupported, they close, occluding flow (termed zone 1). Such a situation does not generally exist in the normal lung but it can be demonstrated in cases where hydrostatic effects are increased, such as a centrifuge [6]. Between these two extremes is a region in which pulmonary arterial pressure exceeds alveolar pressure, but pulmonary venous pressure does not. In this region (zone 2), blood flow is determined not by the difference between arterial and venous pressures, but by the difference between arterial and alveolar pressures. Each capillary acts as a Starling resistor. As a direct consequence, there is a profound vertical gradient in blood flow in zone 2 as while arterial pressure falls with height, alveolar pressure does not. This concept is not new and is probably taught in every pulmonary physiology course in any medical school. It is, however, interesting to recall that until the late 1950s, when the first measurements of regional pulmonary blood flow could be made using radioactive tracers, that the idea was not even appreciated [7].

\section{The Slinky}

The second conceptual idea that is useful is that of the Slinky, a compliant, edge-wound spring in which many children (and adults) delight. Although not a perfect model, the behaviour of this spring is in many respects analogous to that of the lung. If the spring is somewhat stretched (fig. 1b), then the coils at the top of the spring are far apart and those at the bottom close together, a function of the self-weight of the spring on itself. This is analogous to alveolar size, with alveoli at the top of the lung being bigger than those at the bottom. If the string is stretched more (mimicking inspiration), the coils are now more uniformly distributed due to a dominance of the elastic recoil forces of the spring and the degree to which the coils move apart in the lower part of the spring is relatively greater than that in the upper part (and so, by analogy, ventilation is greater in the more dependent lung). Finally, if one imagines blood as flowing through the material of the spring itself, then a bulk observation of blood flow would show a greater blood flow in the dependent portion of the spring, even though the blood flow per coil element is the same [8]. If the effects of gravity are removed (fig. 1c), then these effects are absent and this simple model would predict uniform alveolar size, ventilation and perfusion. 


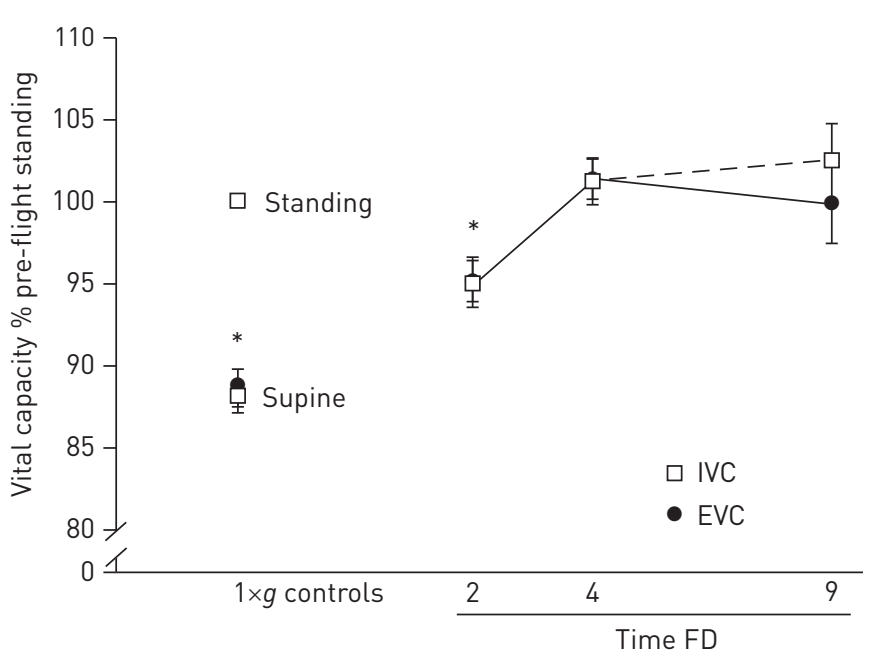

FIGURE 2 Inspiratory vital capacity (IVC) and expiratory vital capacity (EVC) measured over a 9-day exposure to microgravity. There was an initial reduction in vital capacity $\sim 24 \mathrm{~h}$ into the flight (flight day (FD)-2) to a value intermediate to that between standing and supine, and which subsequently returned to that measured pre-flight in the standing posture. ${ }^{\star}: \mathrm{p}<0.05$. Reproduced from [11] with permission from the publisher.

\section{The lung in microgravity}

For the most part, the results presented here were obtained from studies in sustained periods of microgravity in orbital spaceflights lasting 1-2 weeks. These data came from a series of spaceflight studies in which the Space Shuttle carried a shirtsleeves-environment laboratory, Spacelab. This provided a normobaric $(\sim 760 \mathrm{mmHg})$, normoxic (inspiratory oxygen fraction 0.21$)$ environment, albeit one with a slightly elevated carbon dioxide tension $\left(\mathrm{PCO}_{2}\right)(2-4 \mathrm{mmHg})$. In cases in which other conditions prevailed, this is noted. All these measurements were supplemented by an extensive period of pre-flight testing, typically at least four sessions spanning a 6-month period. The same protocols were performed using matching equipment, and the measurements performed both standing erect and supine, to provide appropriate control data. In an effort to keep this review short, a brief overview of the key findings is presented here; however, more extensive reviews are available $[9,10]$.

\section{Lung volumes and expiratory flows}

Vital capacity is arguably the most commonly measured parameter of pulmonary function and the measurement suites employed provided multiple measurements. Vital capacity showed an initial small reduction $(\sim 5 \%)$ when first measured after 1 day in microgravity compared with that measured standing in $1 \times g$, but this reduction was short-lived [11]. By flight day 4, vital capacity had returned to pre-flight values and remained unaltered thereafter (fig. 2). The most plausible explanation for this change was the initial translocation of blood from the lower extremities into the thorax early in flight, with a subsequent reduction as plasma volume was reduced $[12,13]$.

Functional residual capacity (FRC) is dependent on the balance of forces between lung recoil and the outward expansion of the thoracic container. Even before measurements were made, there were clear predictions of the effect of removing gravity [14]. Compared with standing, the removal of gravity would be expected to eliminate the inspiratory force generated by the weight of the abdominal contents, and so FRC would be expected to fall. That is indeed what was observed, with FRC falling by $\sim 500 \mathrm{~mL}$, becoming intermediate between that standing and supine [11]. Unlike vital capacity, there was no change in FRC as a function of time spent in microgravity.

Unlike vital capacity or FRC, both of which are known to change with posture, residual volume is very resistant to change, with upright to supine transitions $[15,16]$ and water immersion $[17,18]$ showing little change. However, somewhat surprisingly, residual volume in microgravity was lower than that standing by $310 \mathrm{~mL}$, an 18\% reduction, and lower than that supine by $220 \mathrm{~mL}$ [11]. The likely explanation of this comes from the uniform alveolar expansion that is present only in microgravity. Under gravity, dependent regions of the lung reach their local residual volume before the entire lung does and so gas remains trapped in these regions, while the upper regions do not deflate to the same extent. However, in microgravity, the uniform alveolar expansion permits a more uniform overall emptying of the lung and a lower total residual volume, as shown in figure 3.

No study of pulmonary function in microgravity could be considered complete without performing forced spirometry and this was included as a standard part of the studies. Just like the measurements of vital capacity (fig. 2), forced vital capacity was reduced early in flight and subsequently recovered [19]. When a careful examination of the effort-independent portion of the maximal expiratory flow-volume (MEFV) 

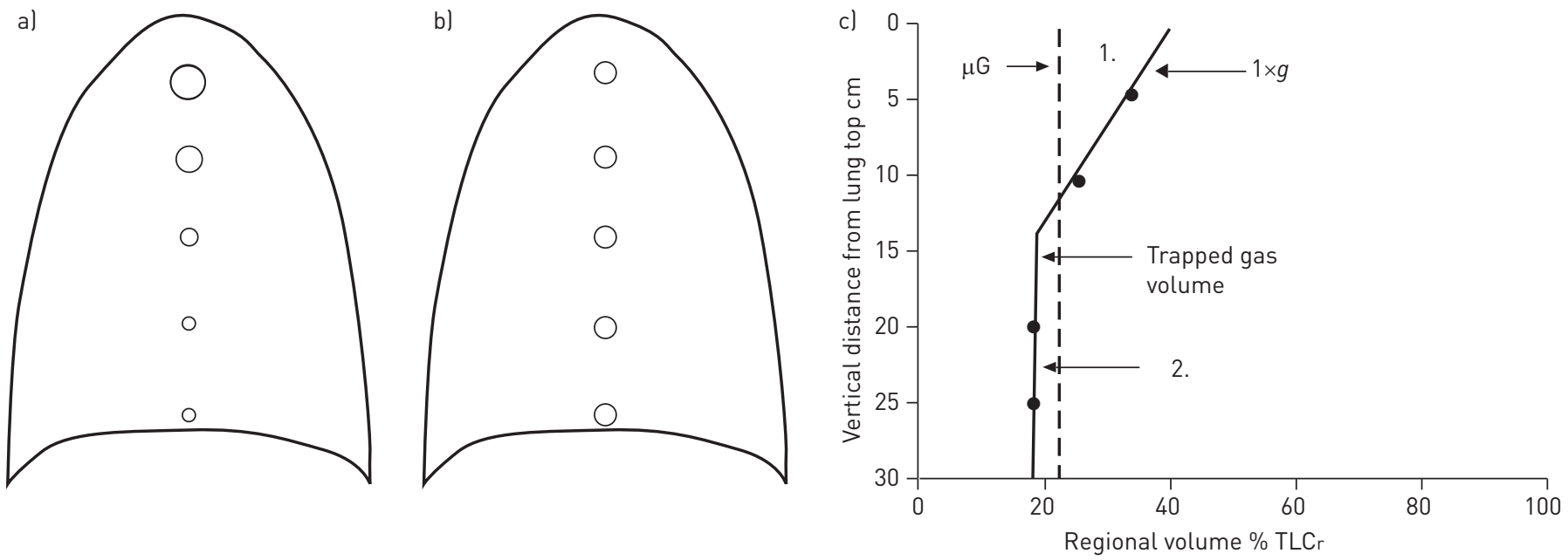

FIGURE 3 A theoretical model of the lung at residual volume in a) $1 \times g$ and b) microgravity $(\mu \mathrm{G})$. c) At residual volume, alveolar size increases from the base of lung to the apex in $1 \times g$ above the point at which airway closure starts, but is uniform in $\mu \mathrm{G}$. If area 2 is less than area 1 , total sum of alveolar volumes will be less in $\mu \mathrm{G}$ than at $1 \times g$. TLCr: regional total lung capacity. Reproduced from [11] with permission from the publisher.

curve was performed, there were changes seen early in flight consistent with increased vascular engorgement that subsequently abated. Such changes had previously been observed in MEFV curves performed in parabolic flight studies [20], a situation in which rapid translocation of blood into the thoracic cavity occurs. In contrast to the parabolic flight results, the changes seen in sustained microgravity were rather small.

Curiously, although there were only modest (or no) changes in virtually all the parameters of forced spirometry, peak expiratory flow was substantially reduced over the first 4 days of flight (by $\sim 12 \%$ before returning to the standing baseline). No clear physiological explanation was found for this and no such reduction was seen in the parabolic flight studies when the subjects were restrained in a seat. The speculation was that subjects had difficulty in achieving maximum flows in the absence of suitable platform against which to brace themselves and that it took some practice before optimal performance could be achieved.

The removal of gravity would be expected to significantly alter chest and abdominal wall mechanics but, unfortunately, no spaceflight studies have been made that included the measurement of oesophageal or gastric pressures necessary for such studies. There was an increase in abdominal contribution to tidal breathing, which rose from $31 \%$ to $58 \%$ in microgravity [21]. This is consistent with results from parabolic flight, in which there was an increase in abdominal wall compliance but not in rib cage compliance [22] consistent with only small changes in chest-wall shape, making for a slightly more circular rib cage $[23,24]$. The changes in shape seem to result from the changes in diaphragm length altering muscle activation [24], a process referred to as operational length compensation [25]. The relatively small effect on the rib cage is also consistent with the relatively small changes in in oesophageal pressure seen in seated subjects in parabolic flight [26]. The studies in parabolic flight had the advantage of measurements both in micro- and hypergravity, and these showed significant nonlinearity in chest wall behaviour $[27,28]$, emphasising the inability to adequately predict the situation in microgravity by extrapolation from hypergravity.

\section{Ventilation}

Much of the knowledge of regional differences in ventilation has come from studies involving imaging [29-31], but the constraints of spaceflight are such that imaging of ventilation has never been performed in orbit. As such, our knowledge is derived from indirect measurements such as single- and multiple-breath wash-outs (or wash-ins) of resident or tracer gases. The typical single-breath wash-out involves a vital capacity inhalation of oxygen and subsequent controlled vital capacity exhalation [32]. During the inspiration, the resident nitrogen is diluted by an amount dependent on the relative regional ventilation, and so nitrogen concentration is now a marker of ventilation. During the exhalation, cardiogenic oscillations are markers of differences in ventilation between lung regions close to and distant from the heart, and the terminal deflection in nitrogen a marker of (in $1 \times g$ ) ventilation differences between dependent and nondependent lung in the presence of airway closure [33]. Inclusion of an argon bolus inhaled at residual volume provides an additional sensitive marker of airway closure. Multiple-breath washouts, in which oxygen is breathed for many breaths, focus on breathing volumes close to the tidal volume and beginning at FRC [34]. Numerous indices are derived from these tests but rather than focus on specific 
values, this review tries to focus the discussion of the results in the bigger picture, referring the reader to specific articles as required.

Based on the aforementioned Slinky model, the expectation would be that pulmonary ventilation should be completely uniform in microgravity. However, when single-breath tests were performed first in parabolic flight [35] and then in spaceflight [36], all of the signatures of ventilatory heterogeneity persisted to some degree. Their continued presence in parabolic flight studies might reasonably have been attributed to the period of hypergravity preceding the microgravity period, but that argument fails in spaceflight studies. The terminal rise in nitrogen concentration (phase IV) in a nitrogen wash-out [33], generally considered a marker of differences in ventilation between the top and bottom of the lung, was greatly reduced in microgravity, to $\sim 20 \%$ (fig. 4 ), which is largely consistent with the Slinky model. This is considered to result from airways reaching their regional closing volume (fig. 3) and, based on the more sensitive data from an argon bolus inhaled at residual volume, the lung volume at which this occurred was the same in microgravity as in $1 \times g$. Thus the overall lung volume at which some lung units reached their point of zero elastic recoil was independent of gravity, a rather surprising result. The persistence of a phase IV is evidence that, independent of gravity, different regions of the lung have different ventilation, perhaps because of differences in regional lung shape.

The second signature of regional differences in ventilation is the cardiogenic oscillations (fig. 4). Contrary to expectations, these persisted at close to $50 \%$ of their size in $1 \times \mathrm{g}$. Indeed, this persistence was noted by the first crew member ever to perform a single-breath test in orbit, who radioed to the ground that the "bumps are still there" as soon as the test was completed. The cardiogenic oscillations result from the physical action of the heart as it expands during diastole on the adjacent lung, and so the persisting oscillations imply differences in ventilation between the lung near the heart and that further away.

The other dominant feature of a single-breath wash-out is the slope of the alveolar plateau, or phase III slope. It is well established that phase III slope increases in early lung disease [37] but the lack of specificity of this change led to it being largely abandoned as a diagnostic test. Unlike the other markers of ventilatory heterogeneity, phase III slope is now known to be largely due to a complex interaction between convective and diffusive processes near the acinar entrance, and critically dependent on the geometry of that lung region (the reason for the high sensitivity of changes in this parameter with early lung disease) [38]. Consistent with this, the phase III slope for nitrogen changed only slightly in microgravity, only falling to $\sim 75 \%$ of that in $1 \times g$.

A subsequent study on a later flight incorporated helium and sulfur hexafluoride into the gas mixtures breathed for the single-breath washouts. These two gases differ widely in molecular weight (4 versus $146 \mathrm{Da}$ ) and so their gas-phase diffusivity differs by a factor of $\sim 6$ (diffusivity scales as the inverse square root of molecular weight). Because of this difference in diffusivity, the interaction with convective flow is different in the lung periphery for these two gases and, as a result, sulfur hexafluoride presents a steeper phase III slope than helium. Curiously, there was a large change in phase III slopes in microgravity; both fell, as was the case for nitrogen, but the changes were such that the helium and sulfur hexafluoride slopes became the same in microgravity, something not seen in $1 \times g$ [39]. Based on these data alone, it was not possible to determine whether the helium slope had dropped less or the sulfur hexafluoride slope dropped more in
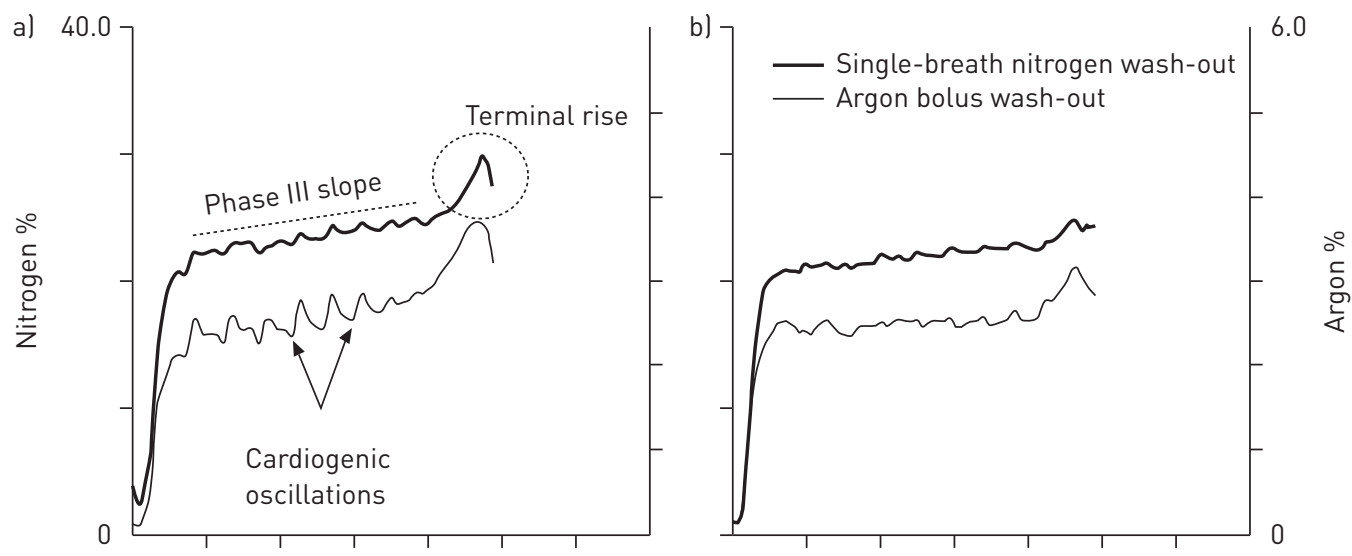

FIGURE 4 Example of the single-breath nitrogen wash-out and argon bolus washout in one subject, standing in a) $1 \times g$ and b) microgravity. The cardiogenic oscillations and terminal rise in concentration are both indicated, as is phase III slope. Reproduced and modified from [36] with permission from the publisher. 
microgravity. However, when the experiment was repeated in parabolic flight, including measurements on one of the same subjects from the spaceflight study, the difference between the slopes persisted, and it was clear that the change had occurred in the behaviour of helium [40]. Since the diffusion-convection interaction for helium occurs at approximately the acinar entrance, the implication is that the geometry of the airways had changed in microgravity. Given the small physical scale of the structures involved, it is hard to imagine a direct gravitational effect causing this in a coordinated manner and the speculation is that there was an accumulation of fluid in the interstitium due to increased capillary filtration, and that this served to generate some peribronchial cuffing in spaceflight. However, no other experiments have yet confirmed or refuted this concept.

The results from the single-breath wash-outs showed a strong persistence of ventilatory heterogeneity and the results from multiple-breath wash-outs, in which gas is washed out over several tidal volume-sized breaths, echoed these results. There were only very modest changes in the indices of these tests (although there were clear increases in heterogeneity in the supine posture) [41]. These results were matched by an innovative analysis of rebreathing data [42], which reached a similar conclusion, namely that the primary determinants of ventilatory inhomogeneity during tidal breathing in the upright posture were not primarily gravitational in origin. Thus, it seems that the elastic properties of the lung dominate gravitational effects during tidal breathing.

\section{Blood flow}

As the lung receives virtually the entire cardiac output, it provides a useful window into cardiac function, something that has been exploited extensively [43-45]. In summary, cardiac output is elevated (compared with standing) by $\sim 35 \%$ after 1 day in microgravity due to a large (60-70\%) increase in stroke volume and a concomitant bradycardia. Cardiac output subsequently falls, presumably as circulating blood volume falls $[12,13]$, but after $\sim 2$ weeks in microgravity, it rises again as the bradycardia seen early in flight abates in the face of a still elevated stroke volume [46]. Interestingly, these changes occur in the face of a reduction in central venous pressure (CVP) $[47,48]$. A thorough explanation of this apparent paradox is still lacking but the implication is that extracardiac pressure must have fallen, which must have occurred as a result of changes in local pressures, as the observed fall in FRC [11] would have implied the opposite.

Unlike cardiac output, which showed adaptive changes with time in microgravity, diffusing capacity for carbon monoxide (DLCO) showed an abrupt and sustained rise [43,44]. DLCO rose by $28 \%$ above that measured standing when measured by the standard single-breath technique [43] and was substantially higher than that measured supine. When measured by a rebreathing technique [44], the results were qualitatively similar. Following return to $1 \times g$, DLCO rapidly returned to pre-flight levels.

The components of the DLCO, membrane diffusing capacity $(D \mathrm{~m})$ and pulmonary capillary blood volume $\left(V_{c}\right)$, were measured by performing carbon monoxide uptake measurements at different oxygen tension values, and these both showed similar increases to that seen in the overall measurement. In contrast, the supine posture showed an increase in $V_{\mathrm{c}}$ but no corresponding increase in $D \mathrm{~m}$. The interpretation of this parallel increase in $D \mathrm{~m}$ and $V_{\mathrm{c}}$ was that the lung had transitioned to entirely zone 2 or 3 conditions (there was no way to determine which condition applied, although zone 3 seems likely), and so pulmonary capillaries were now fully recruited. The consequence of this is an increase in $V_{c}$ as all capillaries are now filled, and an increase in $D \mathrm{~m}$ because of an increase in surface area as previously unfilled capillaries now participate in carbon monoxide uptake (fig. 5).

The large head-ward shift in fluid coupled with a previously hypothesised increase in CVP raised speculation in advance of any measurements of pulmonary oedema formation [49]. However, the large increase in DLCO and the fact that it was sustained over the course of $>1$ week in microgravity suggests this did not occur. Furthermore, measurement of pulmonary tissue volume, a measure of extravascular lung water [50], showed no increase early in flight and was reduced by $\sim 25 \%$ after 9 days in microgravity [44]. Thus, it seems that any supposed increase in pulmonary capillary filtration rate from increase cardiac output and recruitment of previously closed capillaries is insufficient to result in pulmonary oedema capable of compromising gas exchange. However, it is worth recalling the aforementioned subtle changes observed in the studies of pulmonary ventilation that were hypothesised to arise from peribronchial cuffing, perhaps due to a modest degree of pulmonary interstitial oedema insufficient to compromise gas exchange. Based on these observations, one might speculate that the overall lung burden of fluid is somewhat higher in microgravity than in $1 \times g$.

If the hypothesised changes in pulmonary blood volume distribution that led to the changes in DLCO are correct, then one must expect that a measurement of the distribution of pulmonary blood flow would be substantially more uniform in microgravity than in $1 \times g$ (be it standing or supine). While direct measurements of this distribution were not practical, an indirect measure based on a single breath was used. 

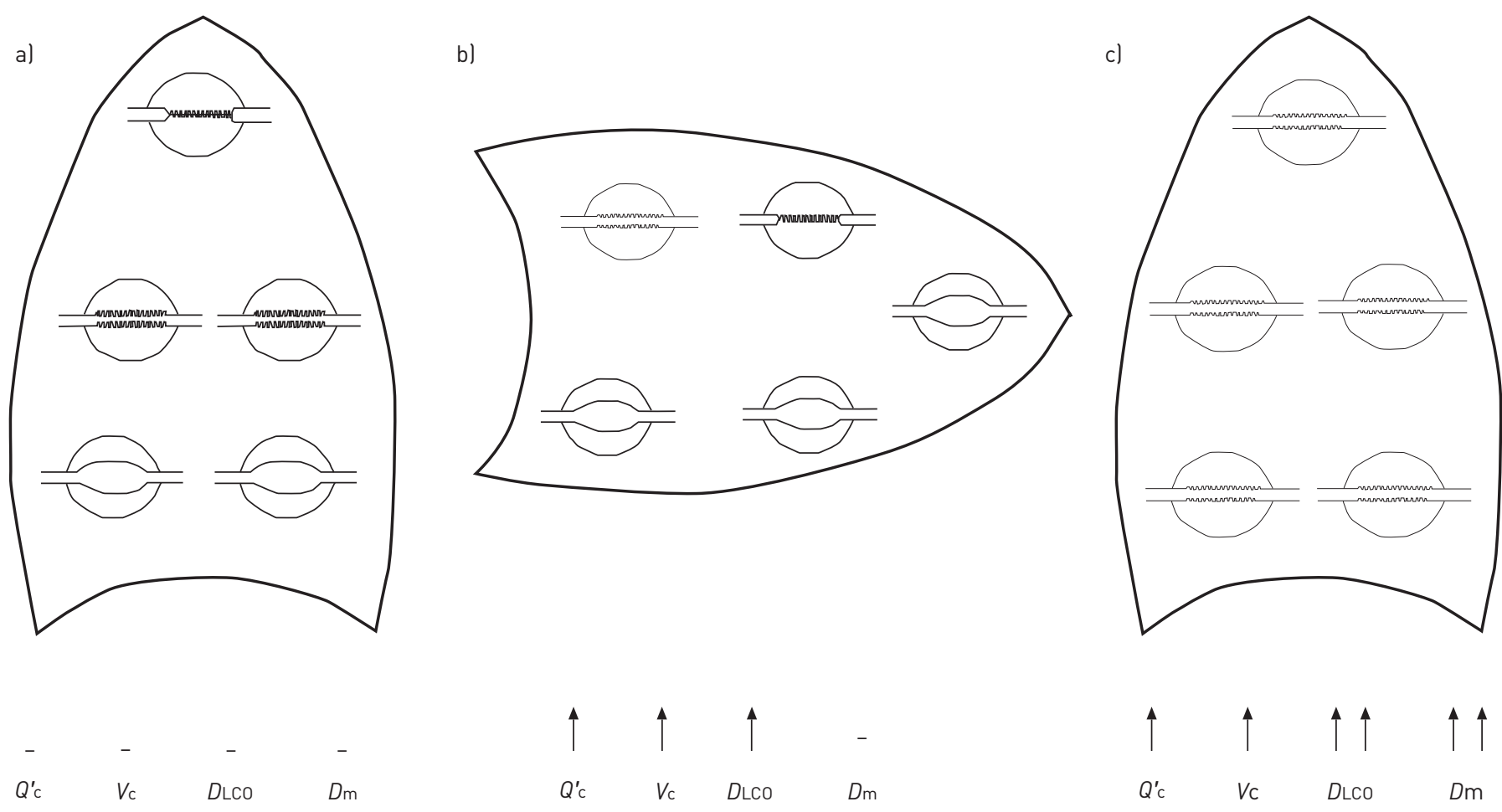

FIGURE 5 The hypothesised basis of the changes in cardiac output $\left(Q^{\prime} c\right)$, membrane diffusing capacity $(D \mathrm{~m})$ and $\left(V_{c}\right)$ that lead to the large increase in diffusing capacity of the lung for carbon monoxide ( $D \mathrm{LCO}$ ) in microgravity. It is unknown whether the lung is in zone 2 or 3 conditions in microgravity but, based on the zone model of pulmonary perfusion, it is expected to be in the same condition throughout. a) Upright position, $1 \times g$, b) supine position, $1 \times g$, c) microgravity. Reproduced from [43] with permission from the publisher.

Subjects hyperventilated to lower $\mathrm{PCO}_{2}$ throughout the lung and then held their breath at total lung capacity (TLC). During this time, carbon dioxide evolves into the alveoli at a rate dependent on regional blood flow (assuming alveolar size is largely uniform at TLC). Thus, the carbon dioxide in the lung is a marker of regional blood flow and a subsequent controlled exhalation acts like the aforementioned single-breath tests used to study the heterogeneity of ventilation. Thus, as with the ventilation studies, the cardiogenic oscillations and the terminal deflection in carbon dioxide are markers of blood flow heterogeneity [35].

The transition from standing to supine showed a reduction in both markers of heterogeneity consistent with a reduction in the vertical extent of the lung with changing posture [51]. Cardiogenic oscillations persisted strongly, suggesting some regional differences in blood flow. However, the complete absence of a terminal deflection (phase IV) in the presence of persisting airways closure (a necessary condition; see the Ventilation section) shows that the regions that close have similar blood flow to those that do not. This is consistent with the abolition of gradients in pulmonary blood flow that result from the zone model (fig. 1a).

\section{Gas exchange and ventilation-perfusion matching}

Since the overall uptake of oxygen and production of carbon dioxide is determined by the metabolic needs of the body, changes in these parameters were expected to be small or even absent, and that indeed was the case [52]. While oxygen consumption and carbon dioxide production were unaltered, there were some alterations in how this was achieved. The principal change was that alveolar ventilation decreased slightly (albeit not quite reaching the level of significance) and end-tidal $\mathrm{PCO}_{2}$ significantly increased by $\sim 2 \mathrm{mmHg}$. This result is somewhat confounded by no increase in one flight and a larger increase in a second flight. These two flights differed in that the cabin $\mathrm{PCO}_{2}$ was higher on the second flight than the first. Subsequent measurements in long-duration spaceflight [53] showed a comparable $\sim 2$-mmHg increase in end-tidal $\mathrm{PCO}_{2}$ but the question of whether this is an effect of the increased cabin carbon dioxide levels or a change in the ventilatory control set-point is unknown.

The breathing pattern leading to the observed alveolar ventilation did, however, change. There was a substantial reduction in resting tidal volume of $\sim 15 \%$ and a concomitant increase in breathing frequency of $\sim 9 \%$, reducing total ventilation by $\sim 7 \%$ [52]. This was accompanied by a reduction in the physiological deadspace, consistent with a more uniform distribution of pulmonary blood flow (see earlier), which resulted in the small reduction in alveolar ventilation. The "selection" of a lower tidal volume and increased 
breathing frequency probably results from the removal of the weight of the abdominal contents and shoulder girdle placing the inspiratory muscles in a different configuration. There was no evidence of significant changes in respiratory drive, with inspiratory time as a fraction of breath length being elevated slightly in microgravity $(\sim 3 \%)$ and average inspiratory flow rate being decreased by $\sim 10 \%$. The opposite direction of these changes in both of the primary measures of respiratory drive suggests that any overall change in resting respiratory drive is small in microgravity.

Just as with ventilation and perfusion (see earlier), direct measurements of the distribution of ventilationperfusion ratio $\left(V^{\prime} \mathrm{A} / Q^{\prime}\right)$ were not practical in spaceflight and it was necessary to rely on an indirect method. The range of $V^{\prime} \mathrm{A} / \mathrm{Q}^{\prime}$ in the lung can be inferred from a single slow exhalation [54-56]. In essence, the respiratory exchange ratio at any point in the exhalation is a reflection of the underlying $V^{\prime} \mathrm{A} / Q^{\prime}$ and so a range of that $V^{\prime} \mathrm{A} / Q^{\prime}$ can be inferred. Given that the degree of heterogeneity of both ventilation and perfusion in the lung were greatly reduced in microgravity (see earlier), a reasonable inference might be that the range of $V^{\prime} \mathrm{A} / Q^{\prime}$ in the lung in $\mu \mathrm{G}$ would be expected to be greatly reduced. This however was not the case. While there was a reduction in the range of $V^{\prime} \mathrm{A} / Q^{\prime}$ seen after the onset of airways closure (phase IV), consistent with the abolition of the top-to-bottom gradients in both ventilation and perfusion, over the majority of the exhalation (phase III, before airway closure) the range of $V^{\prime} \mathrm{A} / Q^{\prime}$ was unchanged. A subsequent examination of the phase relationships of the cardiogenic oscillations provided an explanation [57]. In $1 \times g$, these showed that areas of high ventilation were coincident with areas of high perfusion and areas of low ventilation coincident with areas of low perfusion. The net effect is to make the underlying distributions of ventilation and perfusion correlate with each other, serving to reduce the heterogeneity of the resulting distribution of $V^{\prime} \mathrm{A} / Q^{\prime}$. However, in microgravity, that correlation broke down and so despite reductions in the heterogeneity of ventilation and of perfusion, heterogeneity of $V^{\prime} \mathrm{A} / Q^{\prime}$ was not reduced. Put simply, gravity imposes common effects on both ventilation and perfusion (the zone and Slinky models) serving to maintain a high gas exchange efficiency in the lung.

\section{Ventilatory control and sleep}

Sleep has often been reported to be of poor quality in microgravity [58-60] and one potential contributor might be changes in ventilatory control. Both the hypercapnic and hypoxic ventilatory responses were tested using short rebreathing techniques lasting $\sim 4 \mathrm{~min}$ each. Overall, the carbon dioxide response measured by the READ [61] rebreathing technique, as determined by the ventilation at a $\mathrm{PCO}_{2}$ of $60 \mathrm{mmHg}$, was unchanged by microgravity, although there were slight changes in the slope of the ventilatory response to increasing carbon dioxide [62]. However, the isocapnic hypoxic response as measured by the rebreathing technique of REEBUCK and CAMPBELL [63] showed a substantial reduction in sensitivity in microgravity. The increase in ventilation in response to a drop in arterial oxygen saturation was only $\sim 50 \%$ of that seen standing in $1 \times g$ [62]. However, pre-flight testing performed in the supine posture showed this was not a result of microgravity per se, but rather a result of the abolition of the hydrostatic pressure gradient between the heart and the carotid bodies, the same effect that occurs when lying down. It had previously been shown that increasing blood pressure at the carotid bodies reduces the carotid chemoreceptor response to oxygen via a central nervous system pathway [64-67]. Given that sleep in $1 \times g$ typically occurs lying down, these results suggest that changes in ventilatory control per se are unlikely to contribute to sleep disruption in spaceflight.

Direct polysomnographic measurements of sleep were made in later Shuttle flights. They showed that in this largely normal population (none of the crew studied had significant sleep disordered breathing), there was a reduction in the apnoea-hypopnoea index in microgravity [68] to $<50 \%$ of that seen in $1 \times g$. When electroencephalography-based arousals from sleep were examined, those that could be attributed to respiratory causes (a respiratory event in the $15 \mathrm{~s}$ preceding the arousal) became virtually absent in microgravity; however, the number of arousals from nonrespiratory causes remained unaltered. Overall, microgravity seemed to reduce sleep disordered breathing, probably through the removal of the gravitational effect on the soft tissues of the upper airways. However, the reduction in respiratory-related arousal suggests that the cause of poor sleep in spaceflight is not related to the respiratory system.

\section{Aerosol transport}

It is now well appreciated that the deposition of aerosols from environmental and other sources in the lung creates a health hazard. In the context of spaceflight, this is usually of little consequence as spacecraft cabins are typically well-filtered environments. However, fires aboard spacecraft, as have occurred on Salyut 7 and on Mir [69], produce large amounts of airborne particles. Furthermore, in the context of future exploration of the Moon, Mars and asteroids, exposure to mineral dust is an almost inevitable consequence, as the dust would be tracked into the habitats on spacesuits, as was the case on the Apollo lunar missions. Some mineral dusts are known to be toxic and lunar dust in particular is thought to possess some properties similar to crystalline quartz. Furthermore, these dusts are thought to have highly reactive surfaces due to the 
absence of an atmosphere to permit oxidation [70]. So, while fully oxidised samples have been shown to have only modest toxicity [71,72], the same may not necessarily be true for particles brought into a habitat directly from the lunar surface. As particles between 0.5 and $2 \mu \mathrm{m}$ in size are primarily deposited by sedimentation (a gravitational process), transport and deposition of these particles in a zero- or reducedgravity environment would be expected to be significantly altered.

Unlike the studies described above, all of the data on aerosol transport were obtained in parabolic flight, in which the cabin pressure was somewhat reduced $(\sim 600 \mathrm{mmHg})$, and in which the $g$-level in the aircraft varied from $\sim 1.8 \times g$ to microgravity and back again, with sustained periods of microgravity of $20-25 \mathrm{~s}$. As the processes involved in aerosol transport are principally physical in nature, they have short time constants and the measurements themselves take little time, so the short periods of microgravity (and hypergravity) were adequate for these studies.

The first studies of total deposition examined 2- $\mu \mathrm{m}$ particles and showed a linear increase in deposition as $g$-level increased [73]. However, when a range of particles sizes was examined, it was seen that smaller particles $(1$ and $0.5 \mu \mathrm{m})$ showed disproportionately high deposition [74], with $1-\mu \mathrm{m}$ particles being deposited at more than twice the expected rate. The conclusion drawn was that some form of "enhanced diffusion", probably the result of irreversibility of flow in the branching airway structure, must play a role. Subsequent studies in which boluses of aerosol were inhaled to different lung depths [75-77] and in which small flow reversals were included [78] have suggested this as the most likely cause, with cardiogenic mixing enhancing deposition in a microgravity environment [79].

The overall outcome of these studies is that in a reduced-gravity environment, overall deposition of inhaled aerosols is probably somewhat reduced, but that those particles that deposit do so in different locations in the airway tree compared with the situation in $1 \times g$. For large particles $(\sim 5 \mu \mathrm{m})$, impaction results in increased relative deposition in the central airways, where clearance mechanisms are effective [80], but for smaller particles $(\sim 1 \mu \mathrm{m})$, the suggestion is that alveolar deposition will be increased [81], raising the possibility that these particles will be retained in the lung for a longer period of time, enhancing their toxic potential.

\section{Long-duration microgravity}

The Space Shuttle missions were of limited duration (the longest being $\sim 17$ days) and so were not able to address the question of whether long periods in sustained microgravity further altered lung function. There were hints of some changes after longer periods in microgravity in Skylab [82] (although these were confounded by the hypobaric environment in that vehicle), on the Russian space station Mir [83] and one rather anecdotal report of arterial hypoxaemia [84] in-flight that would suggest alterations in lung function after sustained periods in microgravity.

It was not until the International Space Station (ISS) became operational that we were able to perform studies in long-duration microgravity. Between 2001 and 2003, we were able to study 10 subjects each exposed to 4-6 months of microgravity. Because of the limited capabilities of the fledgling ISS at that time, the studies in microgravity were much more limited than those in the Space Shuttle and were restricted to tests that could be performed breathing only cabin air.

In-flight, the results obtained on the ISS closely matched those from the shorter-duration Space Shuttle flights. Importantly, the indirect measures of the range of $V^{\prime} \mathrm{A} / Q^{\prime}$ in the lung showed no alteration as a function of time in microgravity [53] and there were no changes in lung volumes or in respiratory muscle strength over the course of the flights. In short, it appeared that the lung behaved entirely normally in microgravity once the changes from the $1 \times g$ environment that had already been seen in the shorterduration flights had occurred.

As a side note, there was a concomitant study of the effects of "space walks" (extravehicular activity (EVA)) on the lung. The question was whether the decompression stress caused by moving from the 1-atm ISS environment to the hypobaric spacesuit environment (the US space suit operates at $220 \mathrm{mmHg}$ of $100 \%$ oxygen and the Russian at $290 \mathrm{mmHg}$ of $100 \%$ oxygen) resulted in venous gas emboli that disrupted the distribution of $V^{\prime} \mathrm{A} / Q^{\prime}$ in the lung. The data collected as part of the study of long-duration microgravity exposure provided the baseline and measurements were made the day following EVA (logistic considerations prevented studies on the same day). The hypothesised effect was not observed, suggesting that the denitrogenation protocol that preceded EVA was indeed effective [85] or that microgravity may have protected against venous bubble formation [86]. The study is notable in that it was performed entirely in microgravity, with no reference to ground conditions. 


\section{The lung after microgravity}

Sustained periods of microgravity are known to have profound and lasting influences on numerous organ systems such as bones, muscles and the heart. It does seem that, for the most part, these organ systems "adapt" to the new environment in which there is no need to support the weight of the body (it has no weight in microgravity) or pump blood against gravity in order to maintain cerebral perfusion. While these adaptations to the new environment appear to cause few problems while still in microgravity, space-farers find themselves ill-adapted to the $1 \times g$ environment on return, with postural hypotension, and reductions in bone and muscle mass. While there is a report of a reduction in respiratory muscle strength after longduration spaceflight [83], this was not borne out by subsequent measurements made on the ISS [53].

What then of the lung itself after microgravity exposure? The relatively short-duration flights of the Space Shuttle (1-2 weeks) showed essentially no significant changes in the function of the lung upon return, although it might reasonably be argued that 2 weeks was simply not long enough to see such an effect.

The studies of pulmonary function made during long-duration spaceflight described in the previous section were supplemented by more comprehensive testing performed on the ground pre- and post-flight. The post-flight studies were divided into the early post-flight period (within 1 week of return) and later. Most notable was the complete absence of any significant changes in $>20$ measurements 1 week after return from 4-6 months in microgravity [87]. There were a few relatively minor changes in DLCO and a couple of indices pertaining to peripheral gas mixing in the lung that were present in the week following return, but these had abated after 1 week. Although the exact cause of these minor changes is unknown, the speculation is that they relate to a modest increase in the amount of water in the lung, which serves to slightly alter the geometry of the bronchioles through peribronchial cuffing (see the discussion on helium and sulfur hexafluoride slopes in the Ventilation section). Whatever the cause, the changes seen in the immediate postflight periods were very small and likely physiologically inconsequential.

While a study with almost completely negative results might sound disappointing, the results are, in fact, important in the context of future exploration-class missions, such as those to the Moon or Mars. In such missions, exposure to low gravity or microgravity might be expected to last for even longer periods than a 6-month tour of duty on the ISS before the participants return to Earth. The results suggest that in a normoxic, normobaric environment, lung function is not a concern during or following long-duration future spaceflight exploration missions of $\leqslant 6$ months and probably significantly longer.

\section{Conclusions}

The studies of lung function in microgravity have highlighted the underlying gravitational physiology of the lung. The zone model of pulmonary blood flow and the Slinky model of lung deformation together provide a solid basis for understanding how the lung changes in the absence of gravity, and, as a consequence, how gravity affects lung function. The studies in long-duration microgravity have shown that despite the fact the lung is clearly very sensitive to gravity, changes in gravity do not result in lasting consequences in its function.

\section{Acknowledgements}

The author thanks the substantial collaborative efforts of J.B. West, H.J.B. Guy, A.R. Elliott, C. Darquenne (all University of California San Diego, La Jolla, CA, USA), M. Paiva (Université Libre de Bruxelles, Brussels, Belgium), D. Linnarsson (Karolinska Institutet, Stockholm, Sweden), and the crews of the Space Shuttle and ISS missions.

\section{References}

Wilson TA, Liu S. Effect of acceleration on the chest wall. J Appl Physiol 1994; 76: 1242-1246.

2 Karmali F, Shelhamer M. The dynamics of parabolic flight: flight characteristics and passenger percepts. Acta Astronaut 2008; 63: 594-602.

3 Stone HL, Warren BH, Wager H. The distribution of pulmonary blood flow in human subjects during zero-g. AGARD Conf Proc 1965; 2: 129-148.

West JB. Respiratory physiology - the essentials. Baltimore, Lippincott Williams \& Wilkins, 2012.

West JB, Dollery CT, Naimark A. Distribution of bloodflow in isolated lung: relation to vascular and alveolar pressures. J Appl Physiol 1964; 19: 713-724.

6 Glaister D. The effect of positive centrifugal acceleration upon the distribution of ventilation and perfusion within the human lung, and its relation to pulmonary arterial and intraoesophageal pressures. Proc R Soc Lond B Biol Sci 1967; 168: 311-334.

7 West JB. Pulmonary gas flow and gas exchange. In: West JB, ed. Respiratory physiology: people and ideas. New York, Oxford Press, 1966; pp. 140-196.

8 Hopkins SR, Henderson AC, Levin DL, et al. Vertical gradients in regional lung density and perfusion in the human lung: the Slinky effect. J Appl Physiol 2007; 103: 240-248.

Prisk GK. The lung in space. Clin Chest Med 2005; 26: 415-438.

10 Prisk GK, Paiva M, West JB. Gravity and the lung: lessons from microgravity. New York, Marcel Dekker, 2001.

11 Elliott AR, Prisk GK, Guy HJB, et al. Lung volumes during sustained microgravity on Spacelab SLS-1. J Appl Physiol 1994; 77: 2005-2014. 
12 Alfrey CP, Udden MM, Leach-Huntoon C, et al. Control of red blood cell mass in spaceflight. J Appl Physiol 1996; 81: 98-104.

13 Leach CS, Alfrey CP, Suki WN, et al. Regulation of body fluid compartments during short-term spaceflight. J Appl Physiol 1996; 81: 105-116.

14 Agostoni E, Mead J. Statics of the respiratory system. In: Fenn WO, Rahn H, eds. Handbook of Physiology. Washington, American Physiological Society, 1964; pp. 387-428.

15 Agostoni E, Gurtner G, Torri G, et al. Respiratory mechanics during submersion and negative-pressure breathing. J Appl Physiol 1966; 21: 251-258.

16 Tenney SM. Fluid volume redistribution and thoracic volume changes during recumbency. J Appl Physiol 1959; 14: $129-132$.

17 Buono MJ. Effect of central vascular engorgement and immersion on various lung volumes. J Appl Physiol 1983; 54: 1094-1096.

18 Burki NK. Effects of immersion to water and changes in intrathoracic blood volume on lung function in man. Clin Sci Mol Med 1976; 51: 303-311.

19 Elliott AR, Prisk GK, Guy HJB, et al. Forced expirations and maximum expiratory flow-volume curves during sustained microgravity on SLS-1. J Appl Physiol 1996; 81: 33-43.

20 Guy HJB, Prisk GK, Elliott AR, et al. Maximum expiratory flow-volume curves during short periods of microgravity. J Appl Physiol 1991; 70: 2587-2596.

21 Wantier M, Estenne M, Verbanck S, et al. Chest wall mechanics in sustained microgravity. J Appl Physiol 1998; 84: 2060-2065.

22 Edyvean J, Estenne M, Paiva M, et al. Lung and chest wall mechanics in microgravity. J Appl Physiol 1991; 71: 1956-1966.

23 Michels DB, Friedman PJ, West JB. Radiographic comparison of human lung shape during normal gravity and weightlessness. J Appl Physiol 1979; 47: 851-857.

24 Estenne M, Gorini M, Van Muylem A, et al. Rib cage shape and motion in microgravity. J Appl Physiol 1992; 73 : 946-954.

25 Green M, Mead J, Sears TA. Muscle activity during chest wall restriction and positive pressure breathing in man. Respir Physiol 1978; 35: 283-300.

26 Videbaek R, Norsk P. Atrial distension in humans during microgravity induced by parabolic flights. J Appl Physiol 1997; 83: 1862-1866.

27 Bettinelli D, Kays C, Bailliart O, et al. Effect of gravity and posture on lung mechanics. J Appl Physiol 2002; 93: 2044-2052.

28 Bettinelli D, Kays C, Bailliart O, et al. Effect of gravity on chest wall mechanics. J Appl Physiol 2002; 92: 709-716.

29 Bryan AC, Milic-Emili J, Pengelly D. Effect of gravity on the distribution of pulmonary ventilation. J Appl Physiol 1966; 21: 778-784.

30 Kaneko K, Milic-Emili J, Dolovich MB, et al. Regional distribution of ventilation and perfusion as a function of body positon. J Appl Physiol 1966; 21: 767-777.

31 Milic-Emili J, Henderson JAM, Dolovich MB, et al. Regional distribution of inspired gas in the lung. J Appl Physiol 1966; 21: 749-759.

32 Buist AS, Ross BK. Predicted values for closing volumes using a modified single breath nitrogen test. Am Rev Respir Dis 1973; 107: 744-752.

33 Nichol GM, Michels DB, Guy HJB. Phase v of the single-breath washout test. J Appl Physiol 1982; 52: 34-43.

34 Lewis SM, Evans JW, Jalowayski AA. Continuous distributions of specific ventilation recovered from inert gas washout. J Appl Physiol 1978; 44: 416-423.

35 Michels DB, West JB. Distribution of pulmonary ventilation and perfusion during short periods of weightlessness. J Appl Physiol 1978; 45: 987-998.

36 Guy HJB, Prisk GK, Elliott AR, et al. Inhomogeneity of pulmonary ventilation during sustained microgravity as determined by single-breath washouts. J Appl Physiol 1994; 76: 1719-1729.

37 Buist AS, Vollmer WM, Johnson LR, et al. Does the single-breath $\mathrm{N}_{2}$ test identify the smoker who will develop chronic airflow limitation? Am Rev Respir Dis 1988; 137: 293-301.

38 Paiva M, Engel LA. The anatomical basis for the sloping $\mathrm{N}_{2}$ alveolar plateau. Respir Physiol 1981; 44: 325-337.

39 Prisk GK, Lauzon AM, Verbanck S, et al. Anomalous behavior of helium and sulfur hexafluoride during singlebreath tests in sustained microgravity. J Appl Physiol 1996; 80: 1126-1132.

40 Lauzon AM, Prisk GK, Elliott AR, et al. Paradoxical helium and sulfur hexafluoride single-breath washouts in short-term vs. sustained microgravity. J Appl Physiol 1997; 82: 859-865.

41 Prisk GK, Guy HJB, Elliott AR, et al. Ventilatory inhomogeneity determined from multiple-breath washouts during sustained microgravity on Spacelab SLS-1. J Appl Physiol 1995; 78: 597-607.

42 Verbanck S, Linnarsson D, Prisk GK, et al. Specific ventilation distribution in microgravity. J Appl Physiol 1996; 80: $1458-1465$.

43 Prisk GK, Guy HJB, Elliott AR, et al. Pulmonary diffusing capacity, capillary blood volume and cardiac output during sustained microgravity. J Appl Physiol 1993; 75: 15-26.

44 Verbanck S, Larsson H, Linnarsson D, et al. Pulmonary tissue volume, cardiac output, and diffusing capacity in sustained microgravity. J Appl Physiol 1997; 83: 810-816.

45 Shykoff BE, Farhi LE, Olszowka AJ, et al. Cardiovascular response to submaximal exercise in sustained microgravity. J Appl Physiol 1996; 81: 26-32.

46 Prisk GK, Fine JM, Elliott AR, et al. Effect of $6 \beta$ head-down tilt on cardiopulmonary function: Comparison with microgravity. Aviation Space and Environmental Medicine 2002; 73: 8-16.

47 Buckey JC Jr, Gaffney FA, Lane LD, et al. Central venous pressure in space. J Appl Physiol 1996; 81: 19-25.

48 Foldager N, Andersen TA, Jessen FB, et al. Central venous pressure in humans during microgravity. J Appl Physiol 1996; 81: 408-412.

49 Permutt S. Pulmonary circulation and the distribution of blood and gas in the lungs. Physiology in the space environment. Washington: NAS NRC 1485B; 1967. pp. 38-56.

50 Petrini MF, Peterson BT, Hyde RW. Lung tissue volume and blood flow by rebreathing: Theory. J Appl Physiol 1978; 44: 795-802. 
51 Prisk GK, Guy HJB, Elliott AR, et al. Inhomogeneity of pulmonary perfusion during sustained microgravity on SLS-1. J Appl Physiol 1994; 76: 1730-1738.

52 Prisk GK, Elliott AR, Guy HJB, et al. Pulmonary gas exchange and its determinants during sustained microgravity on Spacelabs SLS-1 and SLS-2. J Appl Physiol 1995; 79: 1290-1298.

53 Prisk GK, Fine JM, Cooper TK, et al. Vital capacity, respiratory muscle strength and pulmonary gas exchange during long-duration exposure to microgravity. J Appl Physiol 2006; 101: 439-447.

54 West JB, Fowler KT, Hugh-Jones P, et al. Measurement of the ventilation-perfusion ratio inequality in the lung by the analysis of a single expirate. Clin Sci 1957; 16: 529-547.

55 Guy HJ, Gaines RA, Hill PM, et al. Computerized noninvasive tests of lung function. A flexible approach using mass spectrometry. Am Rev Respir Dis 1976; 113: 737-744.

56 Prisk GK, Guy HJB, West JB, et al. Validation of measurements of ventilation-to-perfusion ratio inequality in the lung from expired gas. J Appl Physiol 2003; 94: 1186-1192.

57 Lauzon AM, Elliott AR, Paiva M, et al. Cardiogenic oscillation phase relationships during single-breath tests performed in microgravity. J Appl Physiol 1998; 84: 661-668.

58 Frost JD, Shumante WH, Salamy JG, et al. Sleep monitoring: The second manned skylab mission. Aviat Space Environ Med 1976; 47: 372-382.

59 Santy PA. Analysis of sleep on shuttle missions. Aviat Space Environ Med 1988; 59: 1094-1097.

60 Gundel A, Polyakov VV, Zulley J. The alteration of human sleep and circadian rhythms during space flight. J Sleep Res 1997; 6: 1-8.

61 Read DJC. A clinical method for assessing the ventilatory response to carbon dioxide. Aust Ann Med 1967; 16: 20-32.

62 Prisk GK, Elliott AR, West JB. Sustained microgravity reduces the human ventilatory response to hypoxia but not hypercapnia. J Appl Physiol 2000; 88: 1421-1430.

63 Rebuck AS, Campbell EJM. A clinical method for assessing the ventilatory response to hypoxia. Am Rev Respir Dis 1974; 109: 345-350.

64 Heistad D, Abboud F, Mark AL, et al. Interaction of baroreceptor and chemoreceptor reflexes: modulation of the chemoreceptor reflex changes in baroreceptor activity. Trans Assoc Am Phys 1973; 86: 214-225.

65 Heistad D, Abboud F, Mark AL, et al. Interaction of baroreceptor and chemoreceptor reflexes. J Clin Invest 1974; 53: $1226-1236$.

66 Somers VK, Mark AL, Abboud FM. Interaction of baroreceptor and chemoreceptor reflex control of sympathetic nerve activity in normal humans. J Clin Invest 1991; 87: 1953-1957.

67 Heymans C. The part played by vascular presso- and chemo-receptors in respiratory control. Nobel lectures physiology or medicine (1922-1941). Amsterdam, Elsevier, 1965; pp. 460-481.

68 Elliott AR, Shea SA, Dijk DJ, et al. Microgravity reduces sleep-disordered breathing in normal humans. Am J Respir Crit Care Med 2001; 164: 478-485.

69 Burrough B. Dragonfly, NASA and the crisis aboard Mir. New York, HarperCollins, 1998.

70 Linnarsson D, Carpenter J, Fubini B, et al. Toxicity of lunar dust. Planet Space Sci 2012; 74: 57-71.

71 Scully RR, Lam CW, James JT. Estimating safe human exposure levels for lunar dust using benchmark dose modeling of data from inhalation studies in rats. Inhal Toxicol 2013; 25: 785-793.

72 Lam CW, Scully RR, Zhang Y, et al. Toxicity of lunar dust assessed in inhalation-exposed rats. Inhal Toxicol 2013; 25: 661-678

73 Hoffman RA, Billingham J. Effect of altered G levels on deposition of particulates in the human respiratory tract. J Appl Physiol 1975; 38: 955-960.

74 Darquenne C, Paiva M, West JB, et al. Effect of microgravity and hypergravity on deposition of 0.5- to 3- $\mu \mathrm{m}$ diameter aerosol in the human lung. J Appl Physiol 1997; 83: 2029-2036.

75 Darquenne C, West JB, Prisk GK. Deposition and dispersion of $1 \mu \mathrm{m}$ aerosol boluses in the human lung: effect of micro- and hypergravity. J Appl Physiol 1998; 85: 1252-1259.

76 Darquenne C, West JB, Prisk GK. Dispersion of $0.5-2 \mu \mathrm{m}$ aerosol in micro- and hypergravity as a probe of convective inhomogeneity in the human lung. J Appl Physiol 1999; 86: 1402-1409.

77 Darquenne C, Paiva M, Prisk GK. Effect of gravity on aerosol dispersion and deposition in the human lung after periods of breath-holding. J Appl Physiol 2000; 89: 1787-1792.

78 Darquenne C, Prisk GK. Effect of small flow reversals on aerosol mixing in the alveolar region of the human lung. J Appl Physiol 2004; 97: 2083-2089.

79 Prisk GK, Sa RC, Darquenne C. Cardiogenic mixing increases aerosol deposition in the human lung in the absence of gravity. Acta Astronaut 2013; 92: 15-20.

80 Darquenne C, Zeman KL, Sa RC, et al. Removal of sedimentation decreases relative deposition of coarse particles in the lung periphery. J Appl Physiol 2013; 115: 546-555.

81 Darquenne C, Prisk GK. Particulate deposition in the human lung under lunar habitat conditions. Aviat Space Environ Med 2013; 84: 190-195.

82 Sawin CF, Nicogossian AE, Rummel JA, et al. Pulmonary function evaluation during the skylab and apollo-soyuz missions. Aviat Space Environ Med 1976; 47: 168-172.

83 Baranov VM, Tikhonov NA, Kotov AN. The external respiration and gas exchanges in space missions. Acto Astronautica 1992; 27: 45-50.

84 Haase $\mathrm{H}$, Baranov VM, Asyamolova NM, et al. First results of $\mathrm{PO}_{2}$ examinations in the capillary blood of cosmonauts during a long-term flight in the space station "Mir". 41st Congress of the International Astronautical Federation, Dresden, Germany. Paris, IAF, 1990; pp. 1-4.

85 Prisk GK, Fine JM, Cooper TK, et al. Pulmonary gas exchange is not impaired $24 \mathrm{~h}$ after extravehicular activity. J Appl Physiol 2005; 99: 2233-2238.

86 Karlsson LL, Blogg SL, Lindholm P, et al. Venous gas emboli and exhaled nitric oxide with simulated and actual extravehicular activity. Respir Physiol Neurobiol 2009; 169: Suppl. 1, S59-S62.

87 Prisk G, Fine J, Cooper T, et al. Lung function is unchanged in the $1 \mathrm{~g}$ environment following 6-months exposure to microgravity. Eur J Appl Physiol 2008; 103: 617-623. 